\title{
Advanced Adrenal Imaging: Comparison between Radionuclide and MR Techniques
}

\author{
Maurea $\mathrm{S}^{* 1}$, Mainenti $\mathrm{PP}^{2}$, Ponsiglione $\mathrm{A}^{1}$, and Salvatore $\mathrm{M}^{3}$ \\ ${ }^{1}$ Nuclear Medicine and Radiology, Department of Biomedical Sciences Advanced, University of Naples Federico II \\ (UNINA), Naples, Italy \\ ${ }^{2}$ Institute of Biostructures and Bioimaging (IBB), National Research Council, Naples, Italy \\ ${ }^{3}$ Fondazione SDN (IRCCS), Naples, Italy
}

${ }^{*}$ Corresponding author: Maurea S, Nuclear Medicine and Radiology, Department of Biomedical Sciences Advanced, University of Naples Federico II (UNINA), via Ernesto Murolo n. 5, Naples, Italy, Fax: (39) (81) 5457081, Tel: (39) (81) - 7463560 (2039),E-mail: maurea@unina.it

Citation: Maurea S, Mainenti PP, Ponsiglione A, Salvatore M (2016) Advanced Adrenal Imaging: Comparison between Radionuclide and MR Techniques. J Adv Radiol Med Image 1(1): 103. doi: 10.15744/2456-5504.1.103

Received Date: April 17, 2015 Accepted Date: July 23, 2016 Published Date: July 26, 2016

\begin{abstract}
The aim of this review is to describe the role of nuclear imaging modalities using different radiotracers such as labeled nor-cholesterol, metaiodobenzylguanidine (MIBG) and deoxy-glucose (FDG) in the diagnostic evaluation of patients with adrenal tumours to perform lesion characterization in comparison with MR imaging. We report our experience regarding patients with non-hypersecreting unilateral adrenal masses (incidentalomas), patients with hypersecreting or non-hypersecreting adrenal adenomas, and patients with pheochromocytoma (Pheo). On the basis of our findings, nuclear imaging modalities using specific target agents are able to better characterize, compared with MR, adrenal tumours; in particular, radionuclide techniques are able to identify the nature of adrenal incidentalomas and to differentiate between hypersecreting and non-hypersecreting adenomas as well as between benign and malignant Pheo.
\end{abstract}

Keywords: Adrenals; Tumours; nor-Cholesterol; MIBG; FDG; MRI

\section{Introduction}

The high resolution of anatomic imaging techniques such as computed tomography (CT) and magnetic resonance (MR), used in patients with suspected abdominal diseases, frequently results in detection of unexpected adrenal masses; in such patients, the main clinical question consists of noninvasive differential diagnosis between benign and malignant adrenal lesions in order to select the appropriate treatment [1]. As initial diagnostic approach, clinical and laboratory assessment of cortical and medullary adrenal function allows the identification of hypersecreting adrenal lesions and, hence, the characterization of such tumours [2]. However, a tumour mass may not cause adrenal hyperfunction since it may be non-hypersecreting or secretes non-active products [1-3]. In such conditions, lesion typing remains uncertain. CT and MR accurately provide anatomic details of adrenal tumours and may suggest the nature of such lesions [4-6] in particular, lipid-rich adrenal adenomas may be suggested by CT on the basis of low attenuation coefficient on un-enhanced images and/or early as well as rapid washout on enhanced scans; similarly, MR is able to characterize lipid-rich adenomas using chemical-shift (CS) and/or dynamic-gadolinium sequences.

Nuclear adrenal imaging techniques performed with specifically radiolabeled agents which target elements of adrenal function may provide specific metabolic information for tumour characterization, thus, complementing to morphological imaging modalities [7,8]. In this regard, several radiotracers which display unique biological behaviour may be used in nuclear medicine for adrenal lesion evaluation; these include tracers labelled with single photon emitters such as iodine 131 nor-cholesterol or metaiodobenzylguanidine (MIBG) and, previously, gallium-67 citrate, as well as agents labeled with positron emitters such as carbon-11 hydroxy-ephedrine, fluorine-18 fluoro-deoxy-glucose (FDG), carbon-11 metomidate, and fluorine-18 dihydroxyphenylalanine (DOPA) [7,9-16]. Furthermore, molecular engineering has led to the synthesis of peptides of variable forms and sizes for tumour imaging such as radiolabeled somatostatin analogs that have been proposed in the diagnostic evaluation of malignant adrenal tumours reflecting the presence of somatostatin receptors, such as gallium-68 DOTA or techentium-99m labelled peptides $[17,18]$ however, limited clinical applications of such radiocompounds in patients with adrenal abnormalities are reported. Furthermore, limited comparative studies between radionuclide and MR imaging studied are available [19-21] in particular, a complementary role of MIBG and MR techniques in the diagnostic evaluation of patients with pheochromocytoma (Pheo), has been suggested; however, in these studies no data regarding the imaging characterization of such tumours are reported $[22,23]$. 
In this paper, we describe the role of nuclear imaging using different radiotracers in the diagnostic evaluation of patients with adrenal tumours to perform lesion characterization in comparison with MR images; in particular, we discuss the comparative results of nuclear and MR imaging studies in three different groups of patients with adrenal tumours: 1) patients with incidentalomas, 2) patients with adenomas and 3) patients with Pheo. Imaging studies consisted of MR scans performed using a 3.0 Tesla superconducting magnet scanner (Trio, Siemens Medical Systems, Hoffman Estates, IL); radionuclide techniques consisted of: a) cortical adrenal scintigraphy performed after iodine-131 nor-cholesterol (37 MBq) injection using a large field of view gamma camera (Orbiter, Siemens, Erlangen, Germany); b) medullary adrenal scintigraphy performed after iodine-131 MIBG (37 MBq) injection using a dual head rotating gamma camera (ECAM, Siemens Medical Systems, Hoffman Estates, IL); c) Positron Emission Tomography (PET) performed using a whole-body PET EXACT 47 scanner (Siemens, Erlangen, Germany) after FDG injection (370 Mbq). In Group 1, the criteria to select the radiotracer for adrenal scintigraphy was based on clinical history patient data; in particular, if patients were evaluated during the post-treatment follow-up for neoplastic diseases, FDG PET study was performed to distinguish benign from malignant adrenal lesions; conversely, if patients had no history of malignant tumours, nor-cholesterol and successively MIBG radiopharmaceuticals were employed to respectively identify adenomas and Pheo. In Group 2, cortical adrenal scintigraphy was performed using iodine-131 nor-cholesterol. In Group 3, medullary adrenal scintigraphy was performed using iodine-131 MIBG.

\section{Discussion}

Tumour imaging characterization in patients with adrenal masses is an important topic since diagnostic evaluation currently consists not only of lesion detection, but also of tumour characterization in order to recognize lesion-type, to assess lesion function as well as to differentiate between benign and malignant tumours. Furthermore, imaging modalities are actually advanced as well as technically improved suggesting that qualitative and/or quantitative methods might be helpful for this purpose. In the present paper, we report the results obtained in three different groups of patients with adrenal tumours. On the basis of our findings, nuclear imaging modalities using specific target agents are able to better characterize, compared with MR, adrenal tumours; in particular, radionuclide techniques are able to identify the nature of adrenal incidentalomas and to differentiate between hypersecreting and non-hypersecreting adenomas as well as between benign and malignant Pheo.

\section{Group 1}

The incidence of adrenal nodules incidentally detected has increased as a result of widespread use of imaging techniques such as CT and MRI for the evaluation of the abdomen [1]. In such patients, the main role of imaging is not just to identify the presence of the tumour but to help characterize its type in a non-invasive way; this diagnostic approach will help to ensure appropriate treatment since it is of particular importance to differentiate between benign and malignant lesions. CT and MR studies offer accurate anatomic details of any adrenal tumour and will give an initial analysis of lesion characterization [4-6,24,25]. In particular, adrenal adenomas may be suggested by CT on the basis of low attenuation coefficient on un-enhanced images and/or early as well as rapid washout on enhanced scans. Similarly, MR is able to characterize adenomas using dynamic-gadolinium or chemical-shift (CS) sequences; in this regard, Slapa, et al. [24] used a multifactorial MR analysis evaluating tumour size, T2 (liver) index, CSI ratio and contrast washout that showed high (100\%) diagnostic accuracy to characterize adrenal non-adenomas; furthermore, we also reported similar (93\%) diagnostic accuracy for the same purpose using both MR CS and post-contrast sequences [26].

Pheo also shows specific MR features such as clearly increased signal intensity on T2-weighted images and significant enhancement after gadolinium administration. Since gadolinium-enhanced MR imaging shows a considerable overlap in the characteristics of benign and malignant masses, its clinical applicability is limited in distinguishing adenomatous from non-adenomatous adrenal lesions. Conversely, CS acquisition using the basis of fat content of a lesion can differentiate adenomas from malignant lesions. It is well known that adenomas contain a large amount of cytoplasmatic lipid in contrast to adrenal metastases which contain little or none. In the majority of adenomas, the MR pattern seen consists of a reduction of signal intensity on out-of-phase scan compared with in-phase images, whereas in malignant lesions signal intensity remains unchanged. However, some adenomas may contain insufficient lipid to result in loss of signal on out-of-phase scan as well as malignant primary and secondary adrenal tumours may have lipid component showing loss of signal intensity on CS imaging and therefore this may reduce the accuracy of this technique. Despite MRI effectiveness that was previously reported to correspond to a diagnostic accuracy of $93 \%$ to differentiate between benign and malignant adrenal masses [6], the potential overlap in MR imaging appearances of different tumour type means that there remains a need for complementary functional imaging to characterize non-hypersecreting adrenal masses; for this purpose, radionuclide studies such as nor-cholesterol, MIBG and FDG demonstrated high diagnostic accuracy showing respectively the following values $92 \%, 96 \%$ and $100 \%$ to characterize adenomas, Pheos and malignant adrenal lesions, as previously reported [9].

In particular, radionuclide modalities using specific tracers such as nor-cholesterol, MIBG and FDG may provide in vivo tissue characterization of adrenal tumours being able to differentiate between benign and malignant abnormalities [4,7,9,12-14,2729] these agents have no relation to each other and are taken up by individual parts of adrenals on the basis of entirely separate mechanism and are able to differentiate different types of tumours; in particular, radiolabeled nor-cholesterol scintigraphy allows the characterization of functioning, but not hypersecretory, benign cortical adenomas [9] similarly, MIBG imaging has been demonstrated to be useful to identify non-hypersecreting Pheo While fluoro-18 FDG using PET scanning has been shown to be able to recognize malignant adrenal tumours on the basis of increased glucose metabolism [9]. 
MR qualitative patterns to identify adrenal adenomas consisted of no lesion enhancement after gadolinium or, mainly, signal intensity loss on out-phase CS imaging; however, this latter criterion is more appropriate since no gadolinium enhancement may occur also in other benign adrenal tumours [6,19] conversely, the comparative evaluation of T1-T2 signal intensity changes demonstrated inhomogeneous findings not allowing definite adenoma characterization since on T2 images 54\% of these lesions were hypo- or iso-intense and the other $46 \%$ showed hyperintensity. For Pheo, T2 signal hyper-intensity and significant gadolinium enhancement were both specific occurring in all cases; likewise, in adrenal malignancies signal hyperintensity was found in all cases, while gadolinium enhancement occurred only in $63 \%$ of these tumours. These observations suggest that qualitative T2 signal hyperintensity is not accurate in differentiating adrenal masses. Since CS is not able to identify Pheo or adrenal malignancies, other methodological approaches may be needed. For this purpose, the results of MR quantitative analysis showed that the degree of T2 signal hyperintensity and of lesion enhancement after gadolinium were significantly higher in Pheo compared to those of adenomas and malignant tumours but these latter lesions were not distinguished according to these quantitative criteria [30]. Our findings confirm the data of previous investigations [4,6] suggesting that, although the availability of several technical methods, MR imaging provides only some presumptive criteria for tissue characterization in patients with non-hypersecreting adrenal masses; in particular, signal intensity loss on out-phase CS sequence seems to be the best marker for adenomas and the quantitative assessment of T2 or T1-gadolinium signal intensity allows to better characterize Pheo, while no specific MR criteria for adrenal malignancies are available [30].

Conversely, the results of radionuclide studies were more homogeneous compared to those of MR imaging in terms of adrenal tumour characterization, selectively showing nor-cholesterol uptake only in adenomas, increased MIBG activity only in Pheo and abnormal FDG accumulation only in, both primary and metastatic, adrenal malignancies [30] although a limited group of radionuclide scans were available in our experience, the three different radiotracers that we used were able to identify different types of adrenal masses; in fact, nor-cholesterol uptake occurred in 100\% of adenomas, MIBG concentration was found in $100 \%$ of Pheo, FDG accumulation was observed in 100\% of malignancies, with no collection of false negative or positive results. However, recently a meta-analysis evaluation of FDG PET imaging for adrenal mass characterization by Boland et al. reported a diagnostic sensitivity around 91\% [31] this lower value could be explained considering the wide sample of this meta-analysis. Therefore, radionuclide imaging using specific radiocompounds offers specific non-invasive tissue characterization in patients with nonhypersecreting adrenal masses [12-14,28,29]. In this regard, the selection of the appropriate radioagent to be used depends on the clinical patient history, but it may be limited if appropriate radiopharmaceuticals and nuclear equipment are not available. Because benign adenomas are the most common cause of non-hypersecreting adrenal tumours, labeled nor-cholesterol should be the first choice for patients with no history of cancer disease after un-enhanced CT scan which clearly may detect lipid amount of such lesions. In case of a normal nor-cholesterol scan, MIBG should be used to confirm or rule out the presence of non-hypersecreting Pheo. If MIBG is also normal, FDG PET may be considered when the clinical suspicion of malignancy is high. Conversely, when neoplastic patients are evaluated, FDG PET should be initially performed followed, if normal, by nor-cholesterol and, in sequence, MIBG studies. However, the cost-effectivenes of this approach is not proven and it may not be feasible to perform all these studies in individual patients

Nuclear studies are not routinely used in the diagnostic protocols for managing patients with non-hypersecreting adrenal masses as well as are infrequently considered for clinical decision making in such field [32,33]. Although combinations of anatomic criteria by CT and MR are currently used to identify malignancy or benignancy without resorting to radionuclide imaging studies, a diagnostic role of nuclear imaging may be proposed in this setting which may effect patient management. In fact, a radionuclide diagnosis of non-hypersecreting adenoma by nor-cholesterol requests surgical treatment only if large tumour size occurs, otherwise clinical and imaging follow-up is appropriate; similarly, nuclear characterization of Pheo by MIBG allows to correctly plan surgery with adequate patient preparation, and, finally, early identification of adrenal malignancy by FDG PET may determine timely tumour resection with possible favourable patient prognosis. Thus, in patients with non-hypersecreting adrenal masses MR imaging may provide some presumptive criteria to characterize tumour lesions; no gadolinium enhancement and definite signal intensity loss on CSI suggest adenomas, while quantitatively measured T2-hyperintensity and/or gadolinium enhancement are able to identify Pheo. Conversely, radionuclide techniques offer more specific findings since nor-cholesterol and MIBG uptake occur only in benign lesions such adenoma and Pheo, respectively, and FDG accumulation detects only adrenal malignancies. Therefore, multi-agents adrenal scintigraphy is strongly recommended in the diagnostic protocol of patients with non-hypersecreting adrenal masses, particularly when MR findings are uncertain and inconclusive.

\section{Group 2}

The characterization of adrenal adenomas comparing imaging techniques has been investigated in a previous study [34] using quantitative evaluation of nor-cholesterol uptake, CT attenuation value and CS MR Signal intensity loss, but no comparative data between hypersecreting and non-hypersecreting lesions were reported. Our results comparing the diagnostic accuracy of nor-cholesterol adrenal scintigraphy and that of MR imaging showed that radionuclide scanning using nor-cholesterol and MRI are both able to accurately identify cortical adenomas; however, while semi-quantitative analysis of nor-chol uptake is effective to differentiate between hypersecreting and non-hypersecreting adenomas, MRI signal intensity ratios (SIRs) evaluation is not useful for this purpose [35]. To explain these findings some observations need to be considered; in this regard, the precursor molecule 
from which all adrenocortical steroid hormones are synthesized is cholesterol [36] this ubiquitous substance may be accumulated by adrenal cortex from the circulation via a receptor-mediated pathway for low-density lipoproteins, cholesterol carries that are synthesized in the liver; once incorporated by the adrenal cortex, cholesterol is stored in esterified form and acts as the substrate for enzymatic conversion to various adrenal steroid hormones. These physiological steps represent the biological basis for the use of labeled nor-cholesterol in adrenal cortical scintigraphy; in particular, this radiotracer has been used for clinical purpose to evaluate both hypersecreting and non-hypersecreting adrenal adenomas [37]. Our results confirm previous clinical observation since a high (100\%) diagnostic accuracy of nor-cholesterol imaging occurred in our series; however, the new finding that we found was represented by the significant difference in nor-cholesterol uptake between hypersecreting and non-hypersecreting adenomas on the basis of the semi-quantitative analysis of scintigraphic images that showed a significantly higher nor-cholesterol uptake in hypersecreting lesions compared to those non-hypersecreting; this finding is reasonable since hypersecreting adenomas synthesize and thus secrete more hormones compared to normal adrenal tissue as well as to non-hypersecreting adenomas. However, the significantly higher nor-cholesterol concentration observed in hypersecreting adenomas could depend on larger tumour size of these lesions, but no significant difference in this parameter, measured on MR images, occurred in our series.

What is the meaning of the significantly lower nor-cholesterol uptake that we observed in patients with non-hypersecreting adenomas in our experience? As stated, non-hypersecreting adenomas synthesize and secrete hormones like normal adrenal cortical tissue, however these lesions demonstrated abnormal increased nor-cholesterol uptake, but lower compared to hypersecreting lesions; this finding could complement biochemical evaluation to identify abnormal adrenal function in a non-hypersecreting adenoma, but "functioning" in terms of increased nor-cholesterol uptake; this observation could suggest that in such patients the presence of nor-cholesterol uptake seems to be more sensitive than peripheral hormone levels as funtional index. In this regard, a scintigraphic diagnosis, using labeled nor-cholesterol, of subclinical adrenal disorders has been previously proposed in patients with subclinical Cushing's syndrome when adrenal disease was not yet demonstrable by biochemical methods [38]. Furthermore, semi-quantitative indices of nor-cholesterol uptake in adrenal adenomas have been proposed to be useful in the estimation of adrenal functional degree, especially when hormonal values are not diagnostic as well as during patient follow-up with the aim to identify those lesions that could lead to overt clinical syndromes as a result of hormonal hyperproduction [39,40]. Of note, some adenomas have been shown to produce small amounts of adrenocortical hormones, though insufficient enough to cause clinical symptoms as well as a subclinical autonomous glucocorticoid hypersecretion (SAGH) condition has been identifyed in patients with adenoma, but without symptoms of Cushing syndrome [41,42] in these latter patients, nor-cholesterol imaging with tracer uptake analysis could have a role to deeply investigate the endocrine status of such lesions. Hence, the lower nor-cholesterol uptake might reflect the normal hormone synthesis status of non-hypersecreting adenomas and, thus, regular secretion, but representative of adrenal dysfunction responsible of sub-clinical disorders. However, no follow-up data were available to assess the clinical evolution of adrenal function in our patients; additional follow-up clinical studies are needed to investigate and expand this issue.

The overall data of the comparative analysis demonstrated a similar diagnostic sensitivity of nor-cholesterol nuclear imaging (100\%) and MR scanning (95\%) for the identification and characterization of adrenal cortical adenomas confirming previous studies [7,29] in particular, MR imaging could be preferred to radionuclide imaging since it avoids radiation exposition as well as the only CS sequence could be used in the MR protocol avoiding gadolinium administration. However, when imaging characterization of hypersecreting and non-hypersecreting adenomas was investigated, no specific MR criteria for this purpose were found; in fact, no significant differences in MR SIRs were observed between hypersecreting and non-hypersecreting lesions; these results are likely due to the fact that MR SIRs reflect tissue structural characteristics of adrenal adenomas which are mainly based on intra-lesion fat content and this feature is not related to functional conditions.

\section{Group 3}

Pheo is a chromaffin tissue tumour localized in the adrenal medulla with the specific characteristic to secrete cathecolamines; the most frequent appearance is the sporadic form with unilateral benign involvement of a single gland, but the possibility of bilateral, extra-adrenal, or multiple localization as well as familiar or malignant forms occur in $10 \%$ of cases [43]. In particular, for the World Health Organization Pheo is malignant when metastases occur and the prevalence of malignant dedifferentiation depends on the underlying genetic mutation; the distinction between benign and malignant Pheo based on histological criteria is not easy; in fact, there are no certain histological features to define a lesion as malignant and the only sign of malignancy is the presence of tumour capsule infiltration and/or metastatic lesions in anatomic sites where chromaffin tissue is not otherwise found, such as lymph nodes, liver, lungs, and bone, as well as when vascular invasion is demonstrated.

Tumour imaging characterization in terms of differentiation between benign and malignant lesions is a currently attractive issue in oncology; although benign lesions represent the majority of Pheo, malignancy, as stated may occur in $10 \%$ of patients as well as benign tumours may also rarely show malignant evolution [43]. There are no certain histological criteria to define a lesion as malignant and hence tumour-imaging characterization could be helpful to identify suspicious lesions suitable of unfavorable outcome; for this purpose, no specific CT and/or MR imaging features are available, but these techniques are able only to detect invasive regional tumour growth and/or the presence of distant metastases [4]. Several diagnostic methods, such as biological fluid tests, molecular markers, imaging techniques, and genome studies, have been proposed to differentiate between benign and malignant Pheo $[44,45]$. Previous data suggested that radiolabeled somatostatin analogs might be used to characterize malignant 
Pheo $[18,46]$. On the other hand, MIBG uptake has been demonstrated to reflect the concentration of neurosecretory storage granules in chromaffin tissue tumours [47]. Lesions that concentrate MIBG can be benign or malignant and the ability of MIBG scan for tumour detection depends on both tumour size and differentiation $[7,48]$. Our results demonstrated that quantitative analysis of MIBG uptake may differentiate between benign and malignant Pheo, while MR imaging using signal intensity ratio measurement is not useful for this purpose; in fact, MIBG uptake, measured as tumour lesion OD IR, was significantly higher in malignant lesions compared to benign disease; this finding could depend on larger tumour size of malignant Pheo, but no significant difference in this parameter, measured on MR images, was found [49].

MIBG, a physiological analog of nor-epinephrine and guanethidine, has been shown to undergo specific uptake and storage by adrenal medulla, sympathetic autonomic nervous system and tumours derived from these tissues [50] a significant fraction of MIBG uptake into these tissues is by means of the specific and high affinity sodium as well as energy dependent type I amine uptake mechanism [51]. In particular, storage catecholamine granules have been demonstrated within adrenal medullary tissue and Pheo tumour lesions [47]. Since MIBG uptake reflects the concentration of neurosecretory storage granules in chromaffin normal tissue and in the corresponding tumours, the higher MIBG uptake of lesions in patients with malignancies compared to those of patients with benign tumours, as we observed in our experience [49], suggests a higher concentration of catecholamine in malignant Pheo; thus, this difference could reflect different functional conditions with malignant lesions being more prone to catecholamine secretion crises compared to benign tumours. This observation might explain the severe hypertensive attacks that frequently occur in patients with malignant Pheo and might also justify the use of radiolabeled MIBG for therapeutic purposes in such patients when conventional treatments are not effective $[52,53]$.

Previous comparative studies between MIBG and MR imaging demonstrated the complementary role of these techniques in the diagnostic evaluation of patients with Pheo or paraganglioma [19-21] however, in these studies no data regarding the characterization and differentiation between benign and malignant tumours have been reported. Recently, we demonstrated that in patients with Pheo MIBG uptake is able to differentiate between benign and malignant tumour lesions, while MR is not useful for this purpose [49] in particular, our results showed that there are no specific MR criteria, on both T1- and T2-weighted images, to differentiate between benign and malignant Pheo; in fact, no significant differences in MR signal intensity ratio were observed between tumour lesions of benign and malignant neoplasms; these results are likely due to the fact that MR signal intensity ratio reflects tumour tissue structural characteristics which are not related to metabolic and functional conditions; in this regard, when tumour dedifferentiation occurs in malignant lesions, MIBG fails to be concentrated and conversely tumour abnormalities become FDG avid [54]. Finally, a comparative study between CT, MR and C-11 MTO PET for the evaluation of adrenal incidentalomas has been reported showing that this radiocompound may be useful to characterize adrenal adenomas when CT and MR findings are uncertain [55].

\section{Conclusion}

In conclusion, nuclear imaging modalities using specific target agents are able to better characterize, compared with MR, adrenal tumours; in particular, radionuclide techniques are able to identify the nature of adrenal incidentalomas and to differentiate between hypersecreting and non-hypersecreting adenomas as well as between benign and malignant Pheo. In particular, the selection of the appropriate agent for adrenal scintigraphy depends on clinical patient history and department availability of radiocompounds and equipment; since benign adenomas are the most common cause of non-hypersecreting adrenal tumours, labeled nor-cholesterol should be the first choice for patients with no history of cancer disease after un-enhanced CT scan which clearly may detect lipid amount of such lesions; in case of a normal nor-cholesterol scan, MIBG should be used as second choice to confirm or rule out the presence of non-hypersecreting Pheo; if MIBG is also normal, FDG PET may be considered when the clinical suspicion of malignancy is high. Conversely, when neoplastic patients are evaluated, FDG PET should be initially performed followed, if normal, by nor-cholesterol and, in sequence, MIBG studies.

\section{References}

1. Kloos RT, Gross MD, Francis IR, Korobkin M, Shapiro B (1995) Incidentally discovered adrenal masses. Endocr Rev 16: 460-84.

2. Ross NS, Aron DC (1990) Hormonal evaluation of the patient with an incidentally discovered adrenal mass. N Engl J Med 323: $1401-5$.

3. Gross MD, Shapiro B (1993) Clinical review 50. Clinical silent adrenal masses. J Clin Endocrinol Metab 77: 885-8.

4. Mayo-Smith WW, Boland GW, Noto RB, Lee MJ (2001) State-of-the-art Adrenal Imaging. Radiographics 21: 995-1012.

5. Korobkin M, Brodeur FJ, Francis IR, Quint LE, Dunnick NR, et al. (1998) CT time-attenuation washout curves of adrenal adenomas and nonadenomas. AJR Am J Roentgenol 170: 747-52.

6. Heinz-Peer G, Hönigschnabl S, Schneider B, Niederle B, Kaserer K, et al. (1999) Characterization of adrenal masses using MR imaging with histopathologic correlation. AJR Am J Roentgenol 173: 15-22.

7. Ilias I, Sahdev A, Reznek RH, Grossman AB, Pacak K (2007) The optimal imaging of adrenal tumours: a comparison of different methods. Endocr Relat Cancer 14: 587-99.

8. Lawson MA (2001) Role of molecular imaging in management of nonhypersecreting adrenal masses. J Nucl Med 42: 893-4.

9. Maurea S, Klain M, Mainolfi C, Ziviello M, Salvatore M (2001) The diagnostic role of radionuclide imaging in evaluation of patients with nonhypersecreting adrenal masses. J Nucl Med 42: 884-92. 
10. Truong B, Jolles PR, Mullaney JM (1997) Primary adrenal lymphoma: gallium scintigraphy and correlative imaging. J Nucl Med 38: 1770-1.

11. Shulkin BL, Wieland DM, Schwaiger M, Thompson NW, Francis IR, et al. (1992) PET scanning with hydroxyephedrine: an approach to the localization of pheochromocytoma. J Nucl Med 33: 1125-31.

12. Boland GW, Goldberg MA, Lee MJ, Mayo-Smith WW, Dixon J, et al. (1995) Indeterminate adrenal mass in patients with cancer: evaluation at PET with 2-[F-18]-fluoro-2-deoxy-D-glucose. Radiology 194: 131-4.

13. Erasmus JJ, Patz EF, McAdams HP, Murray JG, Herndon J, et al. (1997) Evaluation of adrenal masses in patients with bronchogenic carcinoma using 18Ffluorodeoxyglucose positron emission tomography. AJR Am J Roentgenol 168: 1357-60.

14. Maurea S, Mainolfi C, Bazzicalupo L, Panico MR, Imparato C (1999) Imaging of adrenal tumors using FDG PET: comparison of benign and malignant lesions. AJR Am J Roentgenol 173: 25-9.

15. Bergström M, Juhlin C, Bonasera TA, Sundin A, Rastad J, et al. (2000) PET imaging of adrenal cortical tumors with the 11beta-hydroxylase tracer 11C-metomidate. J Nucl Med 41: 275-82.

16. Timmers HJ, Chen CC, Carrasquillo JA, Whatley M, Ling A, et al. (2009) Comparison of 18F-fluoro-L-DOPA, 18F-fluoro-deoxyglucose, and 18F-fluorodopamine PET and 123I-MIBG scintigraphy in the localization of pheochromocytoma and paraganglioma. J Clin Endocrinol Metab 94: $4757-67$.

17. Prasad V, Baum RP (2010) Biodistribution of the Ga-68 labeled somatostatin analogue DOTA-NOC in patients with neuroendocrine tumors: characterization of uptake in normal organs and tumor lesions. Q J Nucl Med Mol Imaging 54: 61-7.

18. Maurea S, Lastoria S, Caracò C, Klain M, Varrella P, et al. (1996) The role of radiolabeled somatostatin analogs in adrenal imaging. Nucl Med Biol 23: 677-80.

19. Quint LE, Glazer GM, Francis IR, Shapiro B, Chenevert TL (1987) Pheochromocytoma and paraganglioma: comparison of MR imaging with CT and I-131 MIBG scintigraphy. Radiology 165: 89-93.

20. Maurea S, Cuocolo A, Reynolds JC, Tumeh SS, Begley MG, et al. (1993) Iodine-131-metaiodobenzylguanidine scintigraphy in preoperative and postoperative evaluation of paragangliomas: comparison with CT and MRI. J Nucl Med 34: 173-9.

21. van Gils AP, van Erkel AR, Falke TH, Pauwels EK (1994) Magnetic resonance imaging or metaiodobenzylguanidine scintigraphy for the demonstration of paragangliomas? Correlations and disparities. Eur J Nucl Med 21: 239-53.

22. Gross MD, Shapiro B, Francis IR, Glazer GM, Bree RL, et al. (1994) Scintigraphic evaluation of clinically silent adrenal masses. J Nucl Med 35 : 1145-52.

23. Maurea S, Fiumara G, Pellegrino T, Zampella E, Assante R, et al. (2013) MIBG molecular imaging for evaluating response to chemotherapy in patients with malignant pheochromocytoma: preliminary results. Cancer Imaging 13: 155-61.

24. Slapa RZ, Jakubowski W, Januszewicz A, Kasperlik-Zaluska AA, Dabrowska E, et al. (2000) Discriminatory power of MRI for differentiation of adrenal nonadenomas vs adenomas evaluated by means of ROC analysis: can biopsy be obviated? Eur Radiol 10: 95-104.

25. Caoili EM, Korobkin M, Francis IR, Cohan RH, Platt JF, et al. (2002) Adrenal masses: characterization with combined unenhanced and delayed enhanced CT. Radiology 222: 629-33.

26. Maurea S, Imbriaco M, D’Angelillo M, Mollica C, Camera L, et al. (2006) Diagnostic accuracy of chemical-shift MR imaging to differentiate between adrenal adenomas and non adenoma adrenal lesions. Radiol Med 111: 674-86.

27. Maurea S, Lastoria S, Cuocolo A, Celentano L, Salvatore M (1995) The diagnosis of nonfunctioning pheochromocytoma. The role of I-123 MIBG imaging. Clin Nucl Med 20: 22-4.

28. Barzon L, Scaroni C, Sonino N, Fallo F, Gregianin M, et al. (1998) Incidentally discovered adrenal tumors: endocrine and scintigraphic correlates. J Clin Endocrinol Metab 83: 55-62.

29. Maurea S, Klain M, Caraco C, Ziviello M, Salvatore M (2002) Diagnostic accuracy of radionuclide imaging using 131I nor-cholesterol or meta-iodobenzylguanidine in patients with hypersecreting or non-hypersecreting adrenal tumours. Nucl Med Commun 23: 951-60.

30. Maurea S, Caracò C, Klain M, Mainolfi C, Salvatore M (2004) Imaging characterization of non-hypersecreting adrenal masses. Comparison between MR and radionuclide techniques. Q J Nucl Med Mol Imaging 48: 188-97.

31. Boland GW, Dwamena BA, Jagtiani Sangwaiya M, Goehler AG, Blake MA, et al. (2011) Characterization of adrenal masses by using FDG PET: a systematic review and meta-analysis of diagnostic test performance. Radiology 259: 117-26.

32. Young WF (2000) Management approaches to adrenal incidentalomas. A view from Rochester, Minnesota. Endocrinol Metab Clin North Am $29: 159-85$.

33. Schteingart DE (2000) Management approaches to adrenal incidentalomas. A view from Ann Arbor, Michigan. Endocrinol Metab Clin North Am 29: 127-39.

34. Yoh T, Hosono M, Komeya Y, Im SW, Ashikaga R, et al. (2008) Quantitative evaluation of norcholesterol scintigraphy, CT attenuation value, and chemical-shift MR imaging for characterizing adrenal adenomas. Ann Nucl Med 22: 513-9.

35. Maurea S, Imbriaco M, Mollica C, Pace L, Salvatore M (2011) Quantitative imaging characterization of hypersecreting or nonhypersecreting adrenal adenomas: comparison between iodine-131 norcholesterol uptake and magnetic resonance signal intensity ratios. Nucl Med Comm 32: 535-41.

36. Soffer LJ, Dorfam RJ, Gabrilove JL (1961) The human adrenal gland, Lea \& Febiger, Philadelphia, USA.

37. Shapiro B, Gross MD, Sandler MP (1987) Adrenal scintigraphy revisited: a current status report on radiotracers, clinical utility and correlative imaging. Annual Nucl Medicine, Freeman LM, Weissman HS, Raven Press, New York 19: 193-232.

38. Dominguez-Gadea L, Diez L, Piedrola-Maroto G, Crespo A (1996) Scintigraphic diagnosis of subclinical Cushing's syndrome in patients with adrenal incidentalomas. Nucl Med Comm 17: 29-32.

39. La Cava G, Imperiale A, Olianti C, Gheri GR, Ladu C, et al. (2003) SPECT semiquantitative analysis of adrenocortical (131)I-6 beta iodomethyl-norcholesterol uptake to discriminate subclinical and preclinical functioning adrenal incidentaloma. J Nucl Med 44: 1057-64.

40. Imperiale A, Olianti C, Mannelli M, La Cava G, Pupi A (2005) Tomographic evaluation of [131I] 6beta-iodomethyl-norcholesterol standardised uptake trend in clinically silent monolateral and bilateral adrenocortical incidentalomas. Q J Nucl Med Mol Imaging 49: 287-96.

41. Rosen HN, Swartz SL (1992) Subtle glucocorticoid excess in patients with adrenal incidentaloma. Am J Med 92: 213-6.

42. Mansmann G, Lau J, Balk E, Rothberg M, Miyachi Y, et al. (2004) The clinically inapparent adrenal mass: update in diagnosis and management. Endocrine Reviews 25: 309-40.

43. Pacak K, Eisenhofer G, Ahlman H, Bornstein SR, Gimenez-Roqueplo AP, et al. (2007) Pheochromocytoma: recommendations for clinical practice from the First International Symposium. Nat Clin Pract Endocrinol Metab 3: 92-102.

44. Gao B, Kong F, Xu Z (2008/) Development of differential diagnosis for benign and malignant pheochromocytomas. Int J Urol 15 : 771-7. 
45. Kann PH, Wirkus B, Behr T, Klose KJ, Meyer S (2004) Endosonographic imaging of benign and malignant pheochromocytoma. J Clin Endocrinol Metab 89: 1694-7.

46. van der Harst E, de Herder WW, Bruining HA, Bonjer HJ, de Krijger RR, et al. (2001) [(123)I] metaiodobenzylguanidine and [(111)In] octreotide uptake in benign and malignant pheochromocytomas. J Clin Endocrinol Metab 86: 685-93.

47. Bomanji J, Levison DA, Flatman WD, Horne T, Bouloux PM, et al. (1987) Uptake of iodine-123 MIBG by pheochromocytomas, paragangliomas and neuroblastomas: a histopathological comparison. J Nucl Med 28: 973-8.

48. Nguyen HH, Proye CA, Carnaille B, Combermale F, Pattou FN, et al. (1999) Tumour size: the only predictive factor for 131-I MIBG uptake in phaeochromocytoma and paraganglioma. Austr New Zeal J Surg 69: 350-3.

49. Maurea S, Cuocolo A, Imbriaco M, Pellegrino T, Fusari M, et al. (2012) Imaging characterization of benign and malignant pheochromocytoma or paraganglioma: comparison between MIBG uptake and MR signal intensity ratio. Ann Nucl Med 26: 670-5.

50. McEwan AJ, Shapiro B, Sisson JC, Beierwaltes WH, Ackery DM (1985) Radioiodobenzylguanidine for the scintigraphic location and therapy of adrenergic tumour s. Sem Nucl Med 15: 132-53.

51. Jacques S, Tobes MC, Sisson JC (1987) Sodium dependency of uptake of nor-epinephrine and m-iodobenzylguanidine into cultured human pheochromocytoma cells: evidence for uptake-one. Cancer Res 47: 3920-8.

52. Loh KC, Fitzgerald PA, Matthay KK, Yeo PP, Price DC (1997) The treatment of malignant pheochromocytoma with iodine-131 metaiodobenzylguanidine (I-131 MIBG): a comprehensive review of 116 reported patients. J Endocrinol Invest 20: 648-58.

53. Rose B, Matthay KK, Price D, Huberty J, Klencke B, et al. (2003) High dose 131-I metaiodobenzylguanidine therapy for 12 patients with malignant pheochromocytoma. Cancer 98: 239-48.

54. Shulkin BL, Thompson NW, Shapiro B, Francis IR, Sisson JC. (1999) Pheochromocytomas: imaging with 2-[fluorine-18]fluoro-2-deoxy-D-glucose PET. Radiology 212: 35-41.

55. Hennings J, Hellmann P, Ahlstrom H, Sundin A (2009) Computed Tomography, Magnetic Resonance Imaging and C-11 Metomidate positron emission tomography for evaluation of adrenal incidentalomas. Eur J Radiol 69: 314-23.

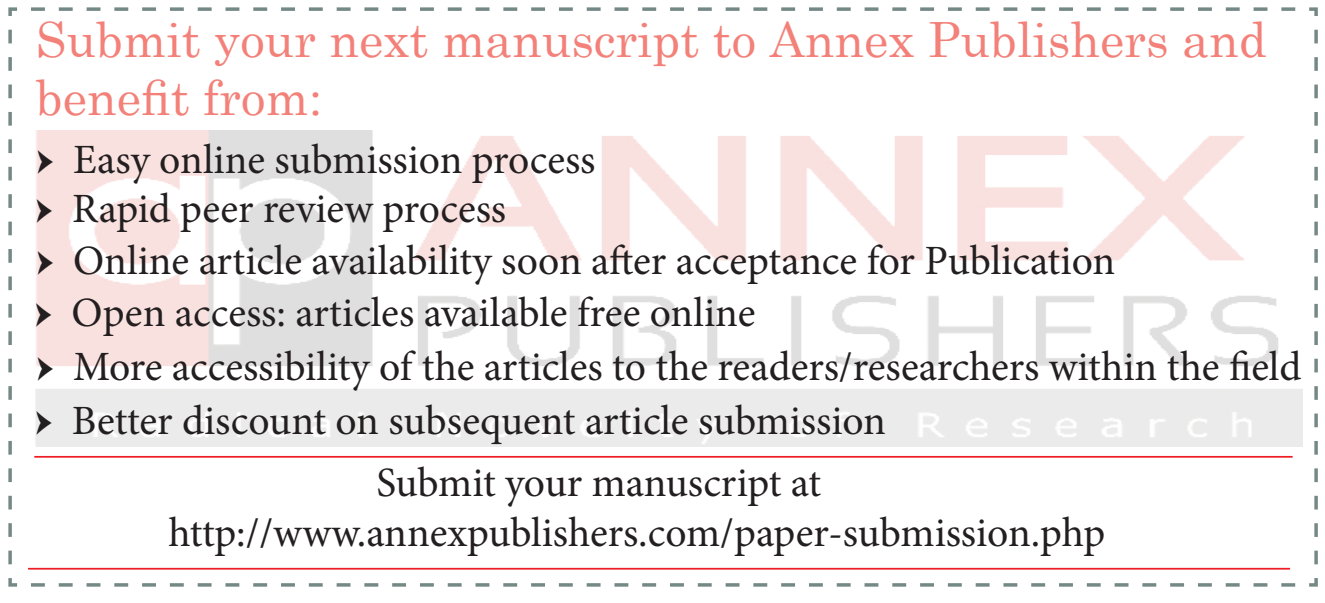

